\title{
Paediatric workload in a multidisciplinary tertiary care intensive care unit in Sri Lanka
}

\author{
S P Mudalige ${ }^{1}$, A N W Karunarathna ${ }^{1}$, I L Wickramanayaka ${ }^{1}$, C D A Goonasekera ${ }^{2}$ \\ Sri Lanka Journal of Child Health, 2009; 38: 92
}

(Key words: Mutidisciplinary paediatric intensive care audit)

\section{Background}

Paediatric intensive care is an emerging speciality in developing countries. In the developed world, paediatric intensive care units (PICUs) concentrate on providing sophisticated technologies and trained personnel to provide special care for this particular age group. PICUs built for such purposes are known to improve the outlook for critically ill children.

In Sri Lanka, there are four PICUs comprising $8 \%$ of the total number of state sector ICUs in the Island ${ }^{1}$. Therefore, most paediatric patients who need intensive care are admitted to multidisciplinary adult ICUs. Data assessing the actual paediatric workload in adult ICU's are scarce.

\section{Objectives}

To assess the paediatric work load and outcomes in terms of mortality and length of ICU stay for critically ill children in a multidisciplinary ICU in a regional hospital.

\section{Method}

This is a retrospective study conducted in the 10 bedded tertiary care ICU in the Teaching Hospital, Peradeniya. Data was extracted from the ICU admission register and death register over a period of 19 months from January 2006 to July 2007. Age below 16 years was considered to be the paediatric group. Data was analyzed using SPSS 10 statistical software.

${ }^{1}$ Temporary Lecturer, ${ }^{2}$ Professor \& Head, Dept. of Anaesthesiology, Faculty of Medicine, University of Peradeniya, Sri Lanka

(Received on 01 September 2008. Accepted on 20 October 2008)

\section{Results}

There were 1107 total admissions to the ICU during the study period, which included 115 (10.4\%) paediatric admissions. Their mean age was 5.38 years (SD 5.06). Of the paediatric admissions, $72.17 \%$ originated from different wards and units of the same hospital and $27.83 \%$ were transferred from elsewhere direct to the ICU. The indications for ICU admission among the paediatric age groups were $43.5 \%$ for post operative observation, $15.6 \%$ for respiratory diseases and $17.4 \%$ for neurological diseases. Of the 115 admissions, $19.13 \%$ were admitted due to sepsis.

The mean duration of ICU stay was 6.65 days (SE 1.64). Of all paediatric admissions, there were 15 deaths $(13 \%)$.

\section{Conclusion}

In a multidisciplinary ICU, 1/10th of the workload relates to the paediatric age group. Their mortality rates appears to be lower (10-15\%) compared to adults $(15-25 \%)$. Of the total ICU beds available in a typical Sri Lankan hospital, 1:10 appear to be a rational proportion of paediatric ICU beds needed.

\section{Acknowledgement}

We thank Mr. Mahes Salgado for his assistance with manuscript preparation.

\section{References}

1. Yatawatta AB, Wanniarachchi CR, Goonasekera CDA. An audit of state sector intensive care services in Sri Lanka. Ceylon Medical Journal 2004; 49(2): 51-4. 
